\title{
Ort der maschinellen Beatmung im Beatmungszentrum - Intensivstation, Intermediate care oder spezialisierte Normalstation
}

\section{B. Schönhofer ${ }^{1}$ \\ T. O. F. Wagner ${ }^{2}$}

\section{Location of Mechanical Ventilation in the Specialized Centre - Intensive Care Unit, Respiratory Intermediate Care Unit and the Specialized Normal Ward}

\section{Zusammenfassung}

Oft sind die beiden Begriffe „Beatmung“ und „Intensivstation“ auch heute noch fest miteinander verknüpft. Die kontinuierlich steigende Anzahl von Beatmungsfällen, der wachsende Kostendruck und die eingeschränkten Ressourcen sind wesentliche Argumente für einen flexiblen Umgang bei der Wahl des Bereiches, in dem beatmet wird. Das pneumologische Beatmungszentrum, in dem sich die Intensivstation für die Maximalversorgung, respiratorische Intermediärstationen und schließlich spezialisierte Normalstationen komplementär ergänzen, hat gute Zukunftsperspektiven. In diesem Konzept kommt der respiratorischen Intermediärstation (respiratory intermediate care unit, RICU) eine Schlüsselrolle zu, da sie einen wichtigen Anteil der bisherigen Aufgaben der Intensivstation effektiv und kostengünstig übernehmen kann. Wichtige Schwerpunkte der RICU sind die schwierige Respiratorentwöhnung und die nicht-invasive Beatmung bei akuter und chronischer Atmungsinsuffizienz. Voraussetzungen für den Therapieerfolg im Bereich der RICU sind das erfahrene Behandlungsteam, adäquate Räumlichkeiten und gute technische Ausstattung.

\section{Abstract}

Until recently "mechanical ventilation" meant "intensive care unit (ICU)". Important arguments for more flexibility concerning the locality where patients are mechanically ventilated are the increase in number of patients, costs and reduced resources. The pulmonary centre for mechanical ventilation, where ICU, respiratory intermediate care unit (RICU) and the specialized normal ward are complementary, is an attractive option for the future. The RICU is the key player in this concept, since as a step down unit it represents a cost-effective approach to the care of substantial numbers of selected patients requiring specialized respiratory care, e.g. intensive respiratory monitoring and therapy, particularly those requiring prolonged mechanical ventilation and non-invasive mechanical ventilation. Success of the RICU requires an experienced team, adequate location and high quality of technical equipment, experienced team, adequate location and high quality of technical equipment.

${ }^{1}$ Abteilung für Pneumologie und internistische Intensivmedizin, Klinikum Region Hannover,

Krankenhaus Oststadt Heidehaus, 30659 Hannover,

${ }^{2}$ Abteilung für Pneumologie und Allergologie, Klinikum der Johann Wolfgang Goethe-Universität, Frankfurt am Main

E-mail: Bernd.Schoenhofer@t-online.de 
Beatmungstherapie beinhaltet heute ein differenziertes Spektrum an Therapieoptionen von der CPAP-Maske als einfachste Form der nicht-invasiven Beatmung (NIV) bis hin zu komplexen Beatmungsformen bei invasiver Beatmung von analgosedierten kritisch Kranken mit Multiorganversagen. Dementsprechend orientiert sich die Therapie an den Erfordernissen des Patienten, wobei Aufwand und Ergebnis in einem möglichst guten Verhältnis stehen sollten. Auf diesem Hintergrund ist es in der Zukunft erforderlich, ein differenziertes Konzept zur Auswahl des Ortes der Beatmung innerhalb einer Institution anzubieten. Hierbei sind die adäquate örtliche Einrichtung und das optimale Beatmungsverfahren vor allem abhängig von der Art und dem Schweregrad der Atmungsstörung.

In diesem Zusammenhang liegt die Zukunft bei den pneumologischen Beatmungszentren, in denen sich die Intensivstation für die Maximalversorgung, respiratorische Intermediärstationen und schließlich spezialisierte Normalstationen komplementär ergänzen. In diesem Konzept kommt der respiratorischen Intermediärstation (respiratory intermediate care unit, RICU) eine Schlüsselrolle zu, da sie einen wichtigen Anteil der bisherigen Aufgaben der Intensivstation effektiv und kostengünstig übernehmen kann.

Bis vor kurzem waren die beiden Begriffe „Beatmung“ und „Intensivstation“ fest miteinander verknüpft. Im klinischen Alltag des Krankenhauses ist es auch heute oft noch so, dass Beatmung ausschließlich auf der Intensivstation stattfindet. Aus mehreren Gründen ist diese fixe Kopplung inzwischen nicht mehr zeitgemäß. Trotz der bestehenden Notwendigkeit, bei Beatmeten eine hohe Qualität in der pflegerischen und ärztlichen Versorgung und des Monitorings zu gewährleisten, sind die kontinuierlich steigende Anzahl von Beatmungsfällen, der wachsende Kostendruck und die eingeschränkten Ressourcen wesentliche Argumente für einen flexibleren Umgang bei der Wahl des Bereiches, in dem beatmet wird. Auch mit zunehmendem Einsatz der NIV und wachsender Anzahl von Entwöhnungseinheiten für langzeitbeatmete Patienten ist die Intensivstation nicht mehr der ausschließliche Ort der maschinellen Beatmung.

Die in diesem Artikel aufgeführten Betrachtungen werden unter besonderer Berücksichtigung eines pneumologischen Beatmungszentrums angestellt. Darunter verstehen wir eine Einrichtung, die in differenzierter Weise und effektiv den Anforderungen moderner Beatmungstherapie unter angemessenem Einsatz von Mitteln für personelle und technische Ausstattung gerecht wird.

\section{Pathophysiologische Betrachtungen}

Zum besseren Verständnis der weiteren Ausführungen zum Ort der Beatmung folgen zunächst einige Vorbemerkungen. Pathophysiologisch lassen sich bei der Atmungsinsuffizienz generell zwei Formen unterscheiden [1]: Erstens die ventilatorische Insuffizienz mit dem Leitwert Hyperkapnie (d.h. erhöhter $\mathrm{PaCO}_{2}$ ) als Ausdruck der überlasteten bzw. erschöpften Atemmuskulatur (Atempumpe) mit sekundärer Hypoxämie und zweitens die pul- monale Insuffizienz mit dem Leitwert Hypoxämie (d.h. verringerter $\mathrm{PaO}_{2}$ ). Daneben sind zur besseren Charakterisierung der Krankheitsbilder Kenntnisse zur zeitlichen Dynamik des Krankheitsverlaufes und zum Schweregrad der Erkrankung erforderlich. Es sind hierbei die chronische Atmungsinsuffizienz bei klinisch stabilen Patienten, die akute Atmungsinsuffizienz (acute respiratory insufficiency, ARI) und die Kombination beider Störungen (d. h. akut auf chronische Insuffizienz, z. B. bei exazerbierter COPD) zu unterscheiden.

\section{Formen der Beatmung in Abhängigkeit vom Krankheitsbild}

Generell stehen zur maschinellen Beatmung (im englischen Sprachgebrauch treffender als „mechanical ventilation“ bezeichnet) als Therapie der Atmungsinsuffizienz invasive und nicht-invasive Beatmungsformen zur Verfügung. Lebensbedrohliche respiratorische Notfälle, wie bei schwergradiger Hypoxämie, aber auch Hyperkapnie mit deutlicher Azidose (d.h. pH<7,20-7,25) bzw. vital bedrohlichem Atemstillstand erfordern in der Regel eine unverzügliche invasive Beatmung. Seit einigen Jahren wird die NIV zunehmend zur Therapie der ARI eingesetzt. Wesentlicher Grund hierfür sind neuere Erkenntnisse zu Risiken und Komplikationen der invasiven maschinellen Beatmung. Hier ist insbesondere die „Tubus-assoziierte Pneumonie“ (auch „Ventilator-assoziierte Pneumonie“, VAP, genannt) von Bedeutung, die mit einer Zunahme der Letalität einhergeht [2]. Die entscheidende Prävention der Tubus-assoziierten Pneumonie besteht daher in der Vermeidung der endotrachealen Intubation, was durch den Einsatz von NIV möglich wird.

Invasive Beatmung an sich ist bereits eine teure Intervention [3]; die Beatmung-assoziierten Komplikationen verbrauchen zusätzlich unverhältnismäßig viele Ressourcen [4]. Die Metaanalyse der randomisierten Studien zur Kosten-Nutzen-Relation von NIV im Vergleich zur Standardtherapie bei ARI ergaben eine Kosteneinsparung von $\$ 2.500$ pro Patientenaufnahme [5]. Hierbei muss allerdings angemerkt werden, dass die einbezogenen Studien eine hohe Heterogenität bzgl. Vergütungssystemen und Eigenschaften der jeweiligen Institutionen in den unterschiedlichen Ländern aufwiesen.

\section{Charakterisierung der Lokalitäten}

Grundsätzlich wird Beatmung im Beatmungszentrum in Abhängigkeit von Form, Schweregrad und zeitlicher Dynamik der Atmungsinsuffizienz in der Intensivstation (intensive care unit, ICU), dem Notfallraum (Emergency room), der respiratorischen Intermediärstation (im Folgenden mit „RICU“ abgekürzt) und schließlich der pneumologischen Normalstation mit Schwerpunkt Beatmungsmedizin durchgeführt. Drei unterschiedliche Betreuungsniveaus des Beatmungszentrums wurden anhand von fünf Haupt- und zwei Nebenkriterien definiert (Tab. 1) [6].

Es gibt zur konkreten Ausgestaltung der räumlichen Konstellation unterschiedliche Konzepte. So wird durchaus kontrovers diskutiert, ob ICU und Intermediate care Station voneinander getrennt oder in einem gemeinsamen Bereich untergebracht werden [7-9]. Da die Realisierung des räumlichen Arrangements 
Tab. 1 Charakterisierung von ICU, RICU und spezialisierten Normalstation [6]

\begin{tabular}{|c|c|c|c|}
\hline & $I C U$ & $\begin{array}{l}\text { Respiratorische } \\
\text { Intermediär- } \\
\text { station }\end{array}$ & $\begin{array}{l}\text { Spezialisierte } \\
\text { Normalstation }\end{array}$ \\
\hline \multicolumn{4}{|l|}{ Hauptkriterien } \\
\hline $\begin{array}{l}\text { Pflege-Patient- } \\
\text { Verhältnis }\end{array}$ & $>3: 1$ & $3: 1-2: 1$ & $<2: 1$ \\
\hline Technik & $\begin{array}{l}\text { polyfunktionales } \\
\text { Monitoring } \\
\text { ICU-Ventilatoren }\end{array}$ & $\begin{array}{l}\text { polyfunktionales } \\
\text { Monitoring } \\
\text { ICU-Ventilatoren } \\
\text { NIV-Ventilatoren }\end{array}$ & $\begin{array}{l}\text { polyfunktionales } \\
\text { Monitoring } \\
\text { NIV-Ventilatoren }\end{array}$ \\
\hline Therapie & $\begin{array}{l}\text { Lungen-Mehror- } \\
\text { ganversagen; Lun- } \\
\text { genversagen mit } \\
\text { schweren Kompli- } \\
\text { kationen }\end{array}$ & $\begin{array}{l}\text { ventilatorische In- } \\
\text { suffizienz } \\
\text { Lungenversagen }\end{array}$ & $\begin{array}{l}\text { ventilatorische In- } \\
\text { suffizienz }\end{array}$ \\
\hline Arzt & $\begin{array}{l}24 \text { h-Schicht-/Be- } \\
\text { reitschaftsdienst }\end{array}$ & Bereitschaft & Bereitschaft \\
\hline Beatmung & $\begin{array}{l}\text { invasive Beat- } \\
\text { mung und NIV }\end{array}$ & $\begin{array}{l}\text { invasive Beat- } \\
\text { mung über Tra- } \\
\text { cheostoma und } \\
\text { NIV }\end{array}$ & $\begin{array}{l}\text { invasive Beat- } \\
\text { mung über Tra- } \\
\text { cheostoma und } \\
\text { NIV }\end{array}$ \\
\hline \multicolumn{4}{|l|}{ Nebenkriterien } \\
\hline Bronchoskopie & sofort verfügbar & sofort verfügbar & Bereitschaft \\
\hline Blutgasanalyse & sofort verfügbar & sofort verfügbar & $\begin{array}{l}\text { binnen } 30 \text { min } \\
\text { verfügbar }\end{array}$ \\
\hline
\end{tabular}

entscheidend von den jeweiligen Gegebenheiten der Institution abhängt, werden die drei Einheiten im Folgenden aus didaktischen Gründen getrennt voneinander abgehandelt.

\section{Die Intensivstation (ICU)}

Unbestritten erfolgt die invasive Beatmung über einen trachealen Tubus beim sedierten bzw. narkotisierten Patienten mit Atmungsinsuffizienz unterschiedlicher Genese im Bereich der ICU. Hier ist die hohe Dichte an Pflegekräften und ärztlichem Personal, aber auch komplexe Technik, z. B. in Form der Intensivventilatoren und des invasiven hämodynamischen Monitoring, vorhanden. Neben internistisch-pneumologischen Krankheitsbildern mit Mehrorganversagen, Sepsis oder hämodynamischer Instabilität ist die invasive Beatmung auf der ICU bei Patienten mit ARI in der postoperativen Phase nach größeren chirurgischen Eingriffen indiziert.

Eingeschränkte Personal- und Bettenkapazitäten und ökonomische Zwänge sind jedoch die wesentlichen Argumente dafür, Beatmung - wenn möglich - außerhalb der ICU durchzuführen. Fehlbelegung der ICU, z.B. durch Patienten mit Notwendigkeit zur Überwachung (z. B. hämodynamisches Monitoring) und aufwändiger Pflege, sind ein alltägliches Problem. Untersuchungen hierzu zeigen, dass in etwa $40 \%$ der ICU-Patienten keine intensivmedizinischen Maßnahmen bzw. invasive Beatmung erforderlich sind $[10,11]$, was zu inadäquatem Verbrauch von Ressourcen der ICU und damit zu unnötiger Kostensteigerung führt.
Die Respiratorische Intermediärstation - zentrale Einheit im Beatmungszentrum

In der pneumologischen Beatmungsmedizin kommt der RICU vor allem mit dem Ziel einer angemessenen Patientenversorgung bei optimaler Kosten-Nutzen-Relation eine wachsende Bedeutung zu. RICU schafft Betten- und Personalkapazität im Bereich der internistischen und pneumologischen, aber auch anästhesiologischen oder interdisziplinären ICU. Die RICU ist Schnittstelle zwischen invasiver Akutbeatmung auf der ICU und anderen stationären Bereichen mit dem Ziel der kompletten Wiederherstellung der Spontanatmung oder einer maschinell unterstützten Spontanatmung.

Da besondere Schwerpunkte der RICU die NIV bei schwergradiger ARI und die schwierige Entwöhnung vom Respirator nach invasiver Langzeitbeatmung sind, wird im Folgenden hierauf etwas ausführlicher eingegangen.

\section{NIV bei schwergradiger ARI}

Während der Initialphase der Therapie mit NIV bei ARI ist der Personalbedarf mit einem Patienten-Therapeuten-Verhältnis von 1:1 relativ hoch. Es ließ sich jedoch zeigen, dass NIV im Vergleich zur konventionellen Therapie (inklusive invasive Beatmung) für ein erfahrenes Team auch während der ersten vier Therapiestunden keinen vermehrten Zeitaufwand bedeutet [12-14]. Im weiteren Verlauf der Behandlung lässt sich dann durch NIV Arbeitszeit und -aufwand einsparen [15].

Generell müssen bei der Wahl des Ortes, an dem NIV zur Behandlung der ARI durchgeführt wird, mehrere Aspekte wie z.B. Schweregrad (z. B. definiert über den pH-Wert) und Genese der ARI, d.h. hyperkapnisch bzw. hypoxämisch und Schulungsstand des Behandlungsteams beachtet werden. Einige prospektive, randomisierte Studien zu NIV bei hyperkapnischer ARI wurden außerhalb der ICU durchgeführt [16-21]. Die Ergebnisse zeigen günstige Tendenzen bzgl. physiologischer Verlaufsparameter und Daten zum Outcome, lassen aber keine allgemeingültigen Schlussfolgerungen zum Stellenwert der NIV außerhalb der ICU zu. Unter dem Einfluss des in Großbritannien herrschenden Mangels an ICU-Betten wurde die bisher größte Studie zum akuten Einsatz der NIV auf Normalstation durchgeführt [21]. In 13 Zentren, die bis dato keine nennenswerte Erfahrung mit NIV hatten, wurden 236 Patienten eingeschlossen. Nach einem einfachen Protokoll wurden die Patienten mit hyperkapnischer ARI in der Interventionsgruppe mit Bilevel-Gerät im Spontanatmungsmodus (d.h. inspiratorische Druckunterstützung) beatmet. Im Vergleich zur Kontrollgruppe wurden durch NIV die Intubationsfrequenz von $27 \%$ auf $15 \%$ und die Mortalitätsrate von $20 \%$ auf $10 \%$ reduziert ( $\mathrm{p}$ jeweils $<0,05$ ). Die Subgruppenanalyse ergab allerdings, dass das Outcome der Patienten mit $\mathrm{pH}<7,3$ während der Initialphase der Beatmung im Vergleich zu Studien, die auf ICU durchgeführt wurden, schlechter war. Die wichtigste Erklärung hierfür sind wahrscheinlich das Fehlen der ICU-assoziierten Qualitätsmerkmale, wie z.B. das Monitoring der Beatmung und Vitalfunktionen, und intensivmedizinische Intervention. Abgesehen von dieser Einschränkung lässt sich aus diesen Studienergebnissen schlussfolgern, dass sich durch frühzeitige Anwendung von NIV auf einer Normalstation das Outcome von Patienten mit hyperkapnischer ARI bei pH-Werten > 7,3 im Ver- 
Tab. 2 Indikationen zur Betreuung auf der RICU

NIV bei hyperkapnischer und hypoxämischer ARI ohne Indikation zur Intubation

- NIV bei Patienten mit schwergradiger ARI, die den Aufenthalt in der ICU, Reanimation und invasive Beatmung ablehnen

- Schwieriges Weaning nach Langzeitbeatmung über Tracheostomie bei kooperationsfähigem Patienten

- Umstellung der invasiven Beatmung von Tracheotomie auf NIV im Weaningprozess

- Verschluss der Tracheotomie bei kritisch kranken Patienten mit Spontanatmung (ggf. mit zwischengeschalteten NIV-Phasen)

- Weiterführung des Monitoring der Atmungsfunktion nach Aufenthalt auf der ICU

- Hustenmanagement mithilfe von NIV, In/Exsufflator, Physiotherapie und Bronchoskopie bei Patienten (vor allem mit neuromuskulären Erkrankungen) mit unzureichendem Hustenstoß zur Verhinderung der Tracheotomie

- Beginn der häuslichen Beatmung bei multimorbidem Patienten und chronisch ventilatorischer Insuffizienz, z. B. mit reduzierter Kooperation oder therapierefraktärer Hypersekretion.

gleich zur konventionellen Therapie ohne Beatmung signifikant bessert. Allerdings ist der einfache Umkehrschluss, nämlich die Normalstation als Ort zur Durchführung der NIV bei Therapie der hyperkapnischen ARI der RICU oder ICU vorzuziehen, nicht allgemein zulässig (z. B. ist das Ausbildungsspektrum der Pflegekräfte bzgl. der Versorgung von schwer Kranken auf Normalstation in den Ländern Europas unterschiedlich). Bezogen auf deutsche Verhältnisse sollte zumindest zum aktuellen Zeitpunkt NIV bei der genannten Indikation im Bereich der RICU durchgeführt werden.

Die Datenlage zum Stellenwert der NIV bei der hypoxämischen ARI ist - im Gegensatz zur hyperkapnischen ARI - nicht eindeutig. Einige Studien zeigten Vorteile der NIV gegenüber der Standardtherapie (incl. Sauerstoffgabe) bei Patienten mit rein hypoxämischer ARI (d.h. $\mathrm{PaO}_{2}<60 \mathrm{~mm} \mathrm{Hg}$ bzw. $\mathrm{SaO}_{2}<90 \%$ bei einer inspiratorischen Sauerstoffkonzentration von $50 \%$ ) $[22,23]$ vor allem aber auch bei gemischt hypoxämisch-hyperkapnischer ARI [24-26]. Demgegenüber liegt die NIV-Versagerquote für ambulant erworbene Pneumonie und ARDS zwischen 30\% und $60 \%$ [27]. Mit der Indikation hypoxämische ARI sollte NIV daher bevorzugt im Bereich der ICU und nur unter optimalen Bedingungen (d.h. räumlich Nähe zu ICU und große Erfahrung des Teams) in der RICU erfolgen, um schwerwiegende Komplikationen (z. B. verzögerte Intubation) zu vermeiden.

\section{Entwöhnung vom Respirator nach Langzeitbeatmung}

Es ist in diesem Zusammenhang sinnvoll, zwischen der unproblematischen Entwöhnung bzw. Extubation und dem Weaningversagen bzw. der schwierigen Entwöhnung zu unterscheiden. In der zuerst genannten Gruppe erfolgt die Extubation unabhängig von der Beatmungsindikation in der Regel nach kurzer Beatmungszeit bereits im Aufwachraum oder auch in der ICU.

Bei der schwierigen Entwöhnung von Respirator und Langzeitbeatmung ist die Sachlage jedoch anders. Hier stellt sich die Frage, ob es angesichts eines effizienten Ressourceneinsatzes noch sinnvoll ist, die Patienten mit Weaningversagen längerfristig im Bereich der ICU zu behandeln. Bei schwer entwöhnbaren Patien- ten, die noch translaryngeal intubiert oder bereits tracheotomiert sind, besteht aufgrund der Komorbidität und/oder langer Beatmungszeiten nach Überwindung der akuten Krise eine protrahierte respiratorische Insuffizienz, die eine weitere mechanische Unterstützung der Ventilation erforderlich macht. Auch wenn hierzu noch keine prospektiv kontrollierten Studien existieren, belegt die praktische Erfahrung, dass mehr als 50\% der Patienten mit Weaningversagen in der RICU eines spezialisierten Zentrums erfolgreich vom Respirator entwöhnt werden können [28]. Die Bedeutung der NIV im Weaningprozess und in der Postextubationsphase wird kontrovers diskutiert. Insbesondere bei beatmeten Patienten mit COPD hat sich NIV im unmittelbaren Anschluss an eine kurzzeitige invasive Beatmung bewährt. Verglichen mit der invasiv beatmeten Kontrollgruppe wurde durch NIV die Entwöhnungsrate verbessert und die Mortalität gesenkt [29]. Ähnlich günstige Ergebnisse mit zusätzlicher Reduktion der Reintubations- und Tracheotomierate durch NIV ließen sich auch bei Patienten nach mehrfach frustran verlaufenden Entwöhnungsversuchen vor der Extubation zeigen [22].

Das besondere Dilemma einer erfolglosen Extubation besteht darin, dass mit der Reintubation eine hohe Nebenwirkungsbzw. Mortalitätsrate verbunden ist [30]. Durch frühzeitigen Einsatz von NIV in der Postextubationsphase ließ sich die Reintubationsrate vor allem bei Risikopatienten mit erneuter ventilatorischer Insuffizienz signifikant reduzieren [31,32]. Ein wesentlicher Grund dafür, dass andere Studien diese Ergebnisse nicht bestätigen, ist der verzögerter Beginn der NIV trotz bestehender Zeichen der ARI $[33,34]$.

Schließlich soll in diesem Zusammenhang erwähnt werden, dass ca. $30 \%$ der Patienten mit neuromuskulären Erkrankungen, Thorakorestriktion und Endstadien der COPD trotz erfolgreichem Weaning vom Respirator nach längerer Spontanatmungsphase eine chronisch ventilatorische Insuffizienz (mit dem Indexparameter „Hyperkapnie“) aufweisen. Diese Patienten lassen sich langfristig durch eine nicht-invasive Heimbeatmung, die vorwiegend in der RICU begonnen wird, stabilisieren [28].

\section{Organisatorische Aspekte und Indikationen}

Die Schulung des Behandlungsteams bzgl. der effektiven Anwendung von NIV ist wesentliche Voraussetzung für den Therapieerfolg und hat daher im Bereich der RICU einen hohen Stellenwert. Insbesondere sind profunde Kenntnisse zu folgenden Aspekten erforderlich: Adaptation von Masken und Kopfgeschirr, technisches Zubehör, Adaptation an Beatmungsgerät, Beatmungsformen, Reinigung und Wartung des technischen Materials, Problemerkennung und -lösung.

Im Vergleich zur ICU gehören zu den wesentlichen Charakteristika der RICU u.a. die aufwändige Pflege und Physiotherapie der Beatmeten, mehr Intimität, längere Besucherzeiten sowie erhaltener Tag-Nacht-Rhythmus (z.B. durch reduzierte Licht- und Lärmbelästigung $[7,8,35])$. Wie Tab.1 zu entnehmen ist, liegen der Personalschlüssel, aber auch das Niveau der technischen Ausstattung und des Monitorings der RICU zwischen ICU und Normalstation.

Wie auch andere Organisationsstrukturen benötigt die RICU eine Mindestgröße (z.B. 6 Beatmungs- bzw. Überwachungsplätze in 
Einzelzimmern), um ökonomisch und funktionell betrachtet effektiv zu sein. Die ärztliche Leitung der RICU sollte die Qualifikationen „Gebietsarzt für Innere Medizin mit Schwerpunkt Pneumologie und Internistische Intensivmedizin“ besitzen. Ein ärztlicher Dienst muss kontinuierlich verfügbar sein. Neben gut geschulten Pflegekräften sind Physio- und Ergotherapeuten sowie Logopäden fester Bestandteil des Behandlungsteams. Weiteres Personal, wie z.B. der Sozialdienst, muss bei Bedarf erreichbar sein.

Die wesentlichen Indikationsbereiche für die RICU sind in Tab. 2 aufgeführt.

\section{Monitoring}

Wesentliches Merkmal der RICU ist das Monitoring der Patienten. Im Gegensatz zur ICU liegt hier der Schwerpunkt allerdings auf nicht-invasiven Verfahren. Bei NIV dient das Monitoring primär der Patientensicherheit und sekundär der Optimierung der Einstellung des Beatmungsgerätes. Der Pflegeschlüssel ermöglicht die Betreuung von maximal drei Patienten durch eine qualifizierte Pflegekraft, wobei besonders die klinische Beobachtung des Patienten (d.h. Erfassung der Vigilanz, der Agitation, Dyspnoe und der Befindlichkeit, Atemfrequenz, Atemanstrengung, Einsatz der Atemhilfsmuskulatur und Atemmuster) wichtig sind $[36,37]$. Mit relativ einfachen Mitteln und ohne weiteres technisches Equipment erfolgt z.B. die Quantifizierung der Dyspnoe [38], die Messung der Atemfrequenz und die Beurteilung des neurologischen Status [39] im Therapieverlauf. Darüber hinaus basiert das nicht-invasive Monitoring der Beatmungsqualität der NIV vorwiegend auf der pulsoximetrisch ermittelten Sauerstoffsättigung $\left(\mathrm{SaO}_{2}\right)$ und der Blutgasanalyse. Problematisch ist die Beurteilung einer formal befriedigenden $\mathrm{SaO}_{2}$ (z. B. > 88\%) häufig jedoch infolge der gleichzeitigen Gabe von Sauerstoff. Vor allem in der Initialphase der NIV muss daher zusätzlich die diskontinuierlich gemessene Blutgasanalyse - und hierbei besonders der pH-Wert und der $\mathrm{PaCO}_{2}$ - in die Beurteilung der Beatmungsqualität einbezogen werden. Sowohl die Entscheidung zur endotrachealen Intubation als auch zur Verlegung des Patienten auf die Intensivstation (z.B. Patienten mit einem pHWert $<7,20-7,25$ ) hängt wesentlich vom Verlauf der BGA-Werte innerhalb der ersten maximal 2 Stunden ab. Optional kann das Monitoring der NIV durch das transkutan oder endexspiratorisch gemessene $\mathrm{CO}_{2}$ ergänzt werden. Schließlich empfiehlt sich das Monitoring der Masken- und Mundleckage, des exspiratorischen Tidalvolumen und der Interaktion zwischen Ventilator und Patient anhand der Druck- und Flusskurven.

\section{Zusätzliches technisches Equipment und Konsiliardienste}

Neben der Beatmungstechnik gehören zur Standardausstattung einer RICU Geräte zur Durchführung internistisch-pneumologischer Diagnostik, wie z.B. Ultraschallgerät für Oberbauchsonographie, Echokardiographie und Gefäßsonographie. Der flexiblen Bronchoskopie kommt hierbei sowohl zur Diagnostik als auch zur Therapie eine besondere Bedeutung zu. Darüber hinaus müssen diagnostische Verfahren, wie z.B. Ösophago-Gastro-Duodenoskopie und Sigmoido- sowie Koloskopie und Radiologie mit CT-Technik kurzfristig verfügbar sein. Gleiches gilt für fachärztliche Konsiliardienste (z.B. Mikrobiologie, HNO, Neurologie, Psychiatrie und Schmerztherapie). Die notwendige Logistik zur Durchführung der Punktionstracheotomie, chirurgischen Tra- cheotomie sowie für den plastischen Verschluss eines Tracheostoma muss gewährleistet sein. Bei Bedarf sollten poly(somno)graphische Diagnostik und Unterstützung durch das Schlaflaborteam im Bereich Masken- und Gerätetechnik möglich sein.

\section{Epidemiologische Daten und Perspektiven}

Mitte der 60-iger Jahre entstanden die ersten RICU in den USA [40]. Auch wenn hierzu bisher keine Evidenz-basierten Daten vorliegen, ermöglicht die RICU im Sinne der „Stepdown unit“ eine optimale Nutzung der ICU für Patienten, die zuvor einer intensivmedizinischen Behandlung bedürfen [41,42]. Umgekehrt wurde nachgewiesen, dass es nach Schließung der RICU zu einem Shift von nicht zwingend intensivpflichtigen Patienten auf die ICU (von $18 \%$ auf $27 \%$ ) und damit verbunden zu einem Kostenzuwachs kam [43]. Die intermediate care unit ist „Kosten-effektiv“. Auch wenn das amerikanische Vergütungssystem nicht ohne weiteres auf unsere Verhältnisse übertragbar ist, zeigten Elpern u. Mitarb., dass sich mit Verlegung von Patienten von der ICU in die RICU die Tageskosten deutlich senken ließen [44]. Eine italienische Studie untersuchte die Effektivität der RICU anhand der Mortalitätsrate in 26 Einheiten mit einem Patienten: Pflegekraft-Verhältnis von $1: 2,5$ bis $1: 4$ pro Schicht mit kontinuierlichem Monitoring, großer Erfahrung des Teams mit NIV, Intubationsbereitschaft und einem ständig verfügbaren Arzt [45]. Die Patientenzuweisung erfolgte zu $47 \%$ aus dem Notfallraum, zu 19\% von internistischen Normalstationen, $18 \%$ aus der ICU, $13 \%$ von pneumologischer Normalstation und 2\% postoperativ. Anhand des Schweregrades der Erkrankung (ermittelt mit APACHE II) ergab sich eine theoretisch zu erwartende (d.h. „predicted“) Mortalität von $22 \%$. Die tatsächliche Mortalität in der RICU lag jedoch mit $16 \%$ signifikant niedriger, was indirekt für die Effektivität der RICU spricht.

Es besteht vielerorts ein Mangel an RICU-Kapazität. Eine diesbezügliche Erhebung in Sheffield, UK ergab, dass nur 26\% von 190 „general hospitals“ über eine RICU verfügten [46]. Andererseits unterscheiden sich organisatorische Struktur und Finanzierungskonzepte von RICU innerhalb Europas, aber auch der einzelnen Staaten $[6,47]$. Es wurden kürzlich die Ergebnisse einer Datenerhebung der Task force der European Respiratory Society (ERS) zu den drei unterschiedlichen Kategorien des Beatmungszentrums (siehe Tab.1) publiziert [6]. Es wurden 68 Einheiten für kritisch Lungenkranke erfasst (d.h. 12 pneumologisch ausgerichtete ICU, 35 RICU und 21 Monitor-Einheiten), wobei deutsche Einrichtungen 27\% ausmachten. Diese Erhebung belegt eindrucksvoll, dass die Pneumologen die Behandlung kritisch Lungenkranker in der RICU trotz fehlender Gesetzesvorgaben und offizieller Ausbildungsprogramme durchführen. Auch wenn die Pneumologie in Europa im Gegensatz zu den USA bisher unzureichend in der Intensivmedizin vertreten ist, sprechen hierfür die in Tab. 3 aufgeführten Argumente [48].

Pneumologische Normalstation mit Schwerpunlkt „NIV in der Heimbeatmung"s

Dieser Bereich ist im Wesentlichen spezialisiert auf die Ersteinstellung und weitere Verlaufskontrolle der Heimbeatmung mit einem geringeren Personalschlüssel als die RICU. Es hat sich als praktikabel erwiesen, dass sich die pneumologische Normalsta- 
Tab. 3 Argumente für den Stellenwert der Pneumologie in der Intensivmedizin ${ }^{48}$

- Die Mehrheit der Einweisungen in die ICU erfolgt aufgrund einer akuten $\mathrm{Er}$ krankung der Lungen mit Störung der Ventilation und des Gasaustausches

- Pneumologen verfügen über profunde Kenntnisse der Lungenphysiologie, insbesondere der Ventilation, der Ventilationsregulation, des Gasaustausches und der kardio-respiratorischen Interaktionen

- Pneumologen sind gleichzeitig auch Internisten

- Pneumologen beherrschen spezielle diagnostische Techniken, wie z.B. Bronchoskopie, die in der Intensivmedizin erforderlich sind

- Pneumologen haben meistens langjährige Erfahrung mit NIV

tion in unmittelbarer Nachbarschaft zum Schlaflabor mit dem Schwerpunkt „Schlafbezogene Atmungsstörungen und nächtliche Hypoventilation" befindet, da gerade in der Adaptationsphase die Beatmung im Schlaf kritisch ist. Neben der Einstellung auf Heimbeatmung liegt eine wesentliche Aufgabe der spezialisierten Normalstationen in der Vorbereitung der Patienten, deren Angehörigen und der ambulanten Pflegekräfte auf die Entlassung des Beatmungspatienten in die häusliche Umgebung, das heimatnahe Krankenhaus bzw. die Pflege- oder Rehabilitationseinrichtung. In mehreren Unterrichtseinheiten müssen Patienten, deren Angehörige, ambulanter Pflegedienst, Hausärzte und zuständige Pneumologen in der Handhabung des Beatmungsgerätes, der Geräteeinstellung, der Erkennung von Gerätedefekten und bzgl. Notfallsituationen geschult werden. Besonders aufwändig ist das Entlassungsmanagement der genannten Gruppen bei tracheotomierten Patienten.

\section{Zielvorgaben für das pneumologische Beatmungszentrum}

Zurzeit ist die Patientenversorgung durch pneumologische Beatmungszentren noch unzureichend. In der Zukunft muss daher eine flächendeckende Versorgung mit RICU und auf Heimbeatmung spezialisierten Normalstationen angestrebt werden. Die Entfernung zum Wohnort des Patienten sollte in der Regel nicht größer als $100 \mathrm{~km}$ sein, damit in der Akutphase die Anbindung des sozialen Umfeldes in den Genesungs- und Entlassungsprozess gewährleistet ist. Hiermit wären auch die i. R. der Verlaufskontrollen der Heimbeatmung erforderlichen An- und Rückfahrten für den chronisch Erkrankten ohne wesentlichen Aufwand realisierbar.

Die diagnostischen und therapeutischen Prozeduren sollten möglichst unter Kriterien des Qualitätsmanagements erfolgen. Die hierzu erforderliche Leitlinien sind von den nationalen und internationalen Gesellschaften (wie z. B. DGP und ERS) zu erstellen.

NIV wird aktuell als vollwertige Beatmungsform alternativ zur invasiven Beatmung im DRG-System geführt. Um diesbezüglich zukunftsfähig zu bleiben, muss eine Spezifizierung der Prozeduren und des therapeutischen Aufwandes bei Anwendung von NIV in der Behandlung der vital bedrohlichen ARI und des schwierigen Weaning erarbeitet werden.
Die wesentlichen Aufgaben der pneumologischen Beatmungszentren bestehen in der Sicherstellung einer qualifizierten Versorgung mit RICU und spezialisierter Pflegestation zur Entwöhnung invasiv langzeitbeatmeter Patienten und ggf. Beginn der häuslichen Beatmung. Teilnahme an Maßnahmen zur Qualitätssicherung, Organisation einer hauseigenen Weiterbildung und Mitarbeit in regionaler und überregionaler Fortbildung sollte in Zukunft verpflichtend sein. Wissenschaftliche Aktivität bevorzugt in multizentrischen Netzwerken und praktische Kooperation mit Haus- und Gebietsärzten, Selbsthilfeverbänden und themenverbundenen Regionalgruppen sind erforderlich.

Ein ambulantes Versorgungsangebot (idealerweise in Form einer integrierten Versorgung) im Netzwerk und in enger Kooperation mit niedergelassenen Pneumologen wäre wünschenswert.

Die spezielle Qualifikation zur „Beatmungspflegekraft“ und „respiratory therapist" wird seit Herbst 2005 in curriculärer Form von der Deutschen Gesellschaft für Pneumologie angeboten [49].

Letztlich ist ein Qualitätsmanagement im Beatmungszentrum unverzichtbar, wobei die Elemente jeder Qualitätssicherung in Form von Definition der Vorgaben und Weiterentwicklung von Struktur-, Verfahrens- und Ergebnisqualität gelten.

\section{Perspektive}

Es ist eine wichtige Aufgabe der involvierten Fachgesellschaften und Krankenhaus- wie Kostenträger, in einer konzertierten Aktion ein funktionstüchtiges Netzwerk zur Versorgung der Patienten mit respiratorischem und/oder ventilatorischem Versagen zu entwickeln.

In Anlehnung an das amerikanische Ausbildungssystem sollte die Intensiv- und Beatmungsmedizin in der Weiterbildungsordnung der Pneumologen verankert werden und fester Bestandteil der pneumologischen Ausbildung in Europa und Deutschland sein.

\section{Literatur}

${ }^{1}$ Schönhofer B, Köhler D. Ventilatorische Insuffizienz und hyperkapnische Kompensation infolge chronisch belasteter „Atempumpe“. Dtsch Med Wochenschr 1994; 119: 1209-1214

2 Fabregas N, Torres A, El-Ebiary M et al. Histopathologic and microbiologic aspects of ventilator-associated pneumonia. Anesthesiology 1996; 84: $760-771$

${ }^{3}$ Dasta JF, McLaughlin TP, Mody SH et al. Daily cost of an intensive care unit day: the contribution of mechanical ventilation. Crit Care Med 2005; 33: $1266-1271$

${ }^{4}$ Rello J, Ollendorf DA, Oster G et al. Epidemiology and outcomes of ventilator-associated pneumonia in a large US database. Chest 2002; 122: $2115-2121$

${ }^{5}$ Keenan SP, Gregor J, Sibbald WJ et al. Noninvasive positive pressure ventilation in the setting of severe, acute exacerbations of chronic obstructive pulmonary disease: more effective and less expensive. Crit Care Med 2000; 28: 2094-2102

${ }^{6}$ Corrado A, Roussos C, Ambrosino N et al. Respiratory intermediate care units: a European survey. Eur Respir J 2002; 20: 1343 - 1350

${ }^{7}$ Nava S, Confalonieri M, Rampulla C. Intermediate respiratory intensive care units in Europe: a European perspective. Thorax 1998; 53: $798-802$ 
${ }^{8}$ Cheng DC, Byrick RJ, Knobel E. Structural models for intermediate care areas. Crit Care Med 1999; 27: 2266-2271

${ }^{9}$ Vincent JL, Burchardi H. Do we need intermediate care units? Intensive Care Med 1999; 25: 1345-1349

${ }^{10}$ Henning RJ, McClish D, Daly B et al. Clinical characteristics and resource utilization of ICU patients: implications for organization of intensive care. Crit Care Med 1987; 15: 264-269

${ }^{11}$ Oye RK, Bellamy PE. Patterns of resource consumption in medical intensive care. Chest 1991; 99: 685-689

12 Bott J, Baudouin SV, Moxham J. Nasal intermittent positive pressure ventilation in the treatment of respiratory failure in obstructive sleep apnoea. Thorax 1991; 46: 457-458

13 Confalonieri M, Potena A, Carbone $G$ et al. Acute respiratory failure in patients with severe community-acquired pneumonia. A prospective randomized evaluation of noninvasive ventilation. Am J Respir Crit Care Med 1999; 160: 1585-1591

${ }^{14}$ Kramer N, Meyer TJ, Meharg J et al. Randomized, prospective trial of noninvasive positive pressure ventilation in acute respiratory failure. Am J Respir Crit Care Med 1995; 151: 1799-1806

${ }^{15}$ Nava S, Evangelisti I, Rampulla C et al. Human and financial costs of noninvasive mechanical ventilation in patients affected by COPD and acute respiratory failure. Chest 1997; 111: 1631 - 1638

${ }^{16}$ Bott J, Carroll MP, Conway JH et al. Randomised controlled trial of nasal ventilation in acute ventilatory failure due to chronic obstructive airways disease. Lancet 1993; 341: $1555-1557$

17 Barbe F, Togores B, Rubi M et al. Noninvasive ventilatory support does not facilitate recovery from acute respiratory failure in chronic obstructive pulmonary disease. Eur Respir J 1996; 9: 1240 - 1245

${ }^{18}$ Angus RM, Ahmed AA, Fenwick LJ et al. Comparison of the acute effects on gas exchange of nasal ventilation and doxapram in exacerbations of chronic obstructive pulmonary disease. Thorax 1996; 51 : $1048-1050$

${ }^{19}$ Wood KA, Lewis L, Harz B von et al. The use of noninvasive positive pressure ventilation in the emergency department: results of a randomized clinical trial. Chest 1998; 113: 1339-1346

${ }^{20}$ Bardi G, Pierotello R, Desideri M et al. Nasal ventilation in COPD exacerbations: early and late results of a prospective, controlled study. Eur Respir J 2000; 15: 98 - 104

${ }^{21}$ Plant PK, Owen JL, Elliott MW. Early use of non-invasive ventilation for acute exacerbations of chronic obstructive pulmonary disease on general respiratory wards: a multicentre randomised controlled trial. Lancet 2000; 355: 1931 - 1935

${ }^{22}$ Ferrer M, Esquinas A, Arancibia F et al. Noninvasive ventilation during persistent weaning failure: a randomized controlled trial. Am J Respir Crit Care Med 2003; 168: 70-76

${ }^{23}$ Hilbert G, Gruson D, Vargas F et al. Noninvasive ventilation in immunosuppressed patients with pulmonary infiltrates, fever, and acute respiratory failure. N Engl J Med 2001; 344: 481 - 487

${ }^{24}$ Wysocki M, Tric L, Wolff MA et al. Noninvasive pressure support ventilation in patients with acute respiratory failure. A randomized comparison with conventional therapy. Chest 1995; 107: 761-768

${ }^{25}$ Martin TJ, Hovis JD, Costantino JP et al. A randomized, prospective evaluation of noninvasive ventilation for acute respiratory failure. Am J Respir Crit Care Med 2000; 161: 807-813

${ }^{26}$ Domenighetti G, Gayer R, Gentilini R. Noninvasive pressure support ventilation in non-COPD patients with acute cardiogenic pulmonary edema and severe community-acquired pneumonia: acute effects and outcome. Intensive Care Med 2002; 28: 1226-1232

${ }^{27}$ Antonelli M, Conti G, Moro ML et al. Predictors of failure of noninvasive positive pressure ventilation in patients with acute hypoxemic re- spiratory failure: a multi-center study. Intensive Care Med 2001; 27: $1718-1728$

${ }^{28}$ Schönhofer B, Euteneuer S, Nava S et al. Survival of mechanically ventilated patients admitted to a specialised weaning centre. Intensive Care Med 2002; 28: 908 - 916

${ }^{29}$ Nava S. Rehabilitation of patients admitted to a respiratory intensive care unit. Arch Phys Med Rehabil 1998; 79: 849-854

${ }^{30}$ Epstein SK. Decision to extubate. Intensive Care Med 2002; 28: $535-546$

${ }^{31}$ Nava S, Gregoretti C, Fanfulla F et al. Noninvasive ventilation to prevent respiratory failure after extubation in high-risk patients. Crit Care Med 2005; 33: 2465-2470

${ }^{32}$ Hilbert G, Gruson D, Portel L et al. Noninvasive pressure support ventilation in COPD patients with postextubation hypercapnic respiratory insufficiency. Eur Respir J 1998; 11: 1349-1353

${ }^{33}$ Keenan SP, Powers C, McCormack DG et al. Noninvasive positive-pressure ventilation for postextubation respiratory distress: a randomized controlled trial. JAMA 2002; 287: 3238-3244

${ }^{34}$ Esteban A, Frutos-Vivar F, Ferguson ND et al. Noninvasive positivepressure ventilation for respiratory failure after extubation. $\mathrm{N}$ Engl J Med 2004; 350: 2452 - 2460

${ }^{35}$ Zimmerman JE, Wagner DP, Knaus WA et al. The use of risk predictions to identify candidates for intermediate care units. Implications for intensive care utilization and cost. Chest 1995; 108: 490-499

${ }^{36}$ Tobin MJ. Respiratory monitoring. JAMA 1990; 264: 244-251

${ }^{37}$ Hess D. Respiratory care devices and techniques through the years. Respir Care 1990; 35: 1234-1237

38 Borg GAV. Psychophysical basis of perceived exertion. Med Sci Sports Exerc 1982; 14: 377-381

${ }^{39}$ Kelly BJ, Matthay MA. Prevalence and severity of neurologic dysfunction in critically ill patients. Influence on need for continued mechanical ventilation. Chest 1993; 104: $1818-1824$

${ }^{40}$ Petty TL, Bigelow DB, Nett LM. The intensive respiratory care unit. An approach to the care of acute respiratory failure. Calif Med 1967; 107: $381-384$

${ }^{41}$ Krieger BP, Ershowsky P, Spivack D. One year's experience with a noninvasively monitored intermediate care unit for pulmonary patients. JAMA 1990; 264: 1143 - 1146

42 Bone RC, Balk RA. Noninvasive respiratory care unit. A cost effective solution for the future. Chest 1988; 93: 390-394

43 Byrick RJ, Mazer CD, Caskennette GM. Closure of an intermediate care unit. Impact on critical care utilization. Chest 1993; 104: 876-881

${ }^{44}$ Elpern EH, Silver MR, Rosen RL et al. The noninvasive respiratory care unit. Patterns of use and financial implications. Chest 1991; 99: 205-208

${ }^{45}$ Confalonieri M, Gorini M, Ambrosino $\mathrm{N}$ et al. Respiratory intensive care units in Italy: a national census and prospective cohort study. Thorax 2001; 56: 373-378

${ }^{46}$ Edbrooke DL, Stevens VG, Hibbert CL et al. High dependency units in England: the lack of provision and the cost of addressing the shortfall. Care Critically Ill 1997; 13: 216-219

47 Schönhofer B. Respiratory high-dependency units in Germany. Monaldi Arch Chest Dis 1999; 54: 448-451

${ }^{48}$ Evans T, Elliott MW, Ranieri M et al. Pulmonary medicine and (adult) critical care medicine in Europe. Eur Respir J 2002; 19: 1202 - 1206

${ }^{49}$ Karg O, Bonnet R, Magnussen $\mathrm{H}$ et al. Respiratory Therapist - Atmungstherapeut - Einführung eines neuen Berufsbildes. Pneumologie 2004; 58: $854-857$ 